\title{
Role of the Prescribing Observatory for Mental Health
}

\author{
Thomas R. E. Barnes and Carol Paton
}

\begin{abstract}
Summary
Positive change in prescribing practice in psychiatric services can be achieved with participation in the UK Prescribing Observatory for Mental Health (POMH-UK) quality improvement programmes. Key elements are feedback of benchmarked performance for local clinical reflection and customised change interventions informed by the national audit findings and parallel qualitative
\end{abstract}

work. However, progress is gradual and gains generally modest.

\section{Declaration of interest}

In the past 3 years, T.R.E.B. has received honoraria for speaking from Lilly and Roche, and C.P. has been a consultant for Roche and Janssen.
Thomas R. E. Barnes (pictured) is a professor of clinical psychiatry in the Centre for Mental Health, Imperial College London, and an honorary consultant at West London Mental Health NHS Trust. Carol Paton is chief pharmacist at Oxleas NHS Foundation Trust and an honorary research fellow in the Centre for Mental Health, Imperial College London. They are the joint-heads of the Prescribing Observatory for Mental Health.

Uniquely among the medical and surgical colleges in the UK, the Royal College of Psychiatrists accommodates a Centre for Quality Improvement. The UK Prescribing Observatory for Mental Health (POMH-UK), based within the Centre for Quality Improvement, was set up in 2005. It was initially funded by a tapering grant from the Health Foundation, but since 2008 has been funded solely through subscriptions from member healthcare organisations. Its aim is to improve the quality of prescribing practice in mental health services. Through focused, audit-based, quality improvement programmes (QIPs), POMH-UK seeks to promote and support the optimal, safest use of existing medications in psychiatric practice.

The QIPs initiated thus far have tackled a range of relatively specific topics. The prescription of high-dose and combined antipsychotics has been addressed in both acute adult in-patient ${ }^{1}$ and forensic settings. Assessment of the side-effects of antipsychotics has been the subject of two QIPs: one targeted at metabolic side-effects in patients cared for by assertive outreach teams, ${ }^{2,3}$ and the other at comprehensive side-effect assessment in patients treated with depot/long-acting injection antipsychotics. Several QIPs have focused on the use of antipsychotic medication for indications or in populations where the supporting evidence base is limited: people with an intellectual disability (referred to as people with learning disability by UK health services) ${ }^{4}$ children and adolescents, and in people with dementia. Other QIPs have covered the use of anti-dementia drugs, ${ }^{5}$ recommended monitoring of lithium treatment, ${ }^{6,7}$ medicines reconciliation at the point of hospital admission ${ }^{8}$ and prescribing for personality disorder. Quality improvement programmes planned for the next couple of years will focus on prescribing for attention-deficit hyperactivity disorder in children and adults, alcohol detoxification in psychiatric services and the use of sodium valproate.

For each QIP, an expert group of experienced clinicians, clinical academics and service users/carers is convened to agree the audit standards: these are usually derived from established, evidence-based clinical guidelines, and their drafting is guided by the principle that they should be accepted by clinicians as undeniable criteria of good care and realistic to achieve in routine clinical practice. The group then develops a bespoke audit tool that is refined at a series of regional workshops attended by staff from member trusts. A baseline audit is then conducted, with the audit data collected by clinicians and clinical audit staff in each participating mental health service and submitted online. The data are analysed at POMH-UK and customised reports are generated for each trust, showing its performance against the audit standards, benchmarked anonymously against the other participating trusts. The performance of individual clinical teams within each trust may be compared with each other, the trust as a whole and the total national sample. Perhaps the most potent element of a QIP is the reflection by clinical teams on their performance. Slide sets that are customised for each participating trust are provided to facilitate local presentation of the data. The data collected allow for the measurement of service adherence to the clinical standards, but also include demographic, diagnostic and other relevant clinical information that provides a context for interpretation of practice, and can inform local strategies and action plans to achieve improvement. Although these audit data are suitable for the purposes of local quality improvement, they are not necessarily appropriate for objective ranking of healthcare organisations. ${ }^{9}$ This is partly because interpretation of an individual trust's performance requires knowledge of the sample selection and the context in which the relevant services are delivered. Further, if used for the purposes of ranking, removal of trust anonymity would be necessary, inevitably undermining the credibility and potency of the benchmarked feedback.

In the time between baseline and re-audit, usually 18 months, change interventions are developed, informed by the baseline audit findings as well as additional questionnaires and qualitative work exploring barriers to, and facilitators of, best practice. The aim of these interventions is to support trusts and clinical teams in closing crucial gaps identified between the prescribing standards and clinical practice. Following re-audit, using the same data-collection tool, a second, customised, benchmarked report on performance is provided, highlighting any changes from baseline at national, trust and clinical-team level. In response to requests from trusts for continued involvement in individual QIPs, supplementary audits have been conducted over subsequent years. Examples of effective interventions by trusts to improve their practice have been shared by reports, and presentations at meetings open to member trusts. Those trusts participating in repeated audits are encouraged to consider to what extent any change in their practice over time is the consequence of implementing local strategies to increase the level of adherence 
to the standards, or reflects other factors, such as the selection of different patient samples at different stages.

\section{What has POMH-UK achieved?}

Year-on-year, POMH-UK membership has grown: the vast majority of UK mental health trusts, as well as several private or charitable healthcare organisations, have joined, and the number participating in each QIP has increased in the past few years to reflect the majority of trusts with relevant services. Indeed, POMH-UK has demonstrated a workable and effective methodology for QIPs in the National Health Service (NHS), and positive changes in clinical practice have been seen in some, although not all, QIPs, along with greater involvement of clinicians and clinical teams in audit and quality improvement processes. The data collected have provided trusts with evidence of the quality of clinical care in the organisation, and supported their submissions showing adherence to national guideline recommendations as part of clinical governance. Detailed information on the quality and variation in national prescribing practice has been made available on topics such as the use of depot/long-acting injection antipsychotics ${ }^{10}$ and for services, such as intellectual disability ${ }^{4}$ and child and adolescent psychiatry, which lack prescribing guidelines and a robust evidence base for pharmacotherapy. The extensive information gathered on national prescribing practice in mental health services has also been used to support the rationale for successful research grant applications. These have included studies of pharmacological strategies that, despite a lack of adequate, formal testing in relation to potential risks and benefits, are commonly used in clinical practice, as shown in the large POMH-UK data-sets, which routinely comprise information on many thousands of prescriptions and may be taken as representative of national practice.

\section{What has POMH-UK learned?}

Trusts may participate in QIPS for various reasons, including using their local audit data for both internal and external benchmarking as a measure of service quality, clinical governance reporting and inclusion in Quality Accounts. Where clinical teams have the opportunity to review their benchmarked performance data, the desire of team members to provide the best care for their patients can be a powerful driver for change, particularly if the audit standards are seen as clearly evidence based, accepted as achievable good practice, and reflect their own clinical priorities. Qualitative work can be helpful in understanding possible barriers to best practice at the level of the system, team and individual patient. It can also identify potential enabling factors, and inform the development of customised change interventions. These range from relatively simple educational tools such as a 'ready reckoner' for calculating total antipsychotic dose (now commonly employed by clinicians in their own prescribing practice and when giving second opinions on pharmacotherapy) and a metabolic syndrome investigations poster, to more complex interventions such as a side-effect information folder, and a patient-held lithium booklet ${ }^{11}$ (developed in collaboration with the National Patient Safety Agency and the National Pharmacy Association).

Any improvements in prescribing practice take time and are generally modest, but incremental and sustained improvement can be achieved over time in individual services. Examples may be drawn from POMH-UK QIPS in relation to side-effect monitoring; substantial improvements were seen in the programmes addressing screening for the metabolic syndrome and side-effect assessment in patients on depot antipsychotics. However, some established healthcare systems, and clinical custom and practice, can be formidable impediments to behavioural change. These may be common to all the QIPS, such as a lack of consistent trust commitment to the process, with slow and incomplete dissemination of audit results throughout participating organisations and little reflection by clinical teams on the content, and the variable uptake of change interventions by services, which are more likely to be adopted if they are seen as relevant to, and compatible with, local practice. Other barriers are specific to particular QIPS, depending on the prescribing issue addressed; for the QIP on high-dose and combined antipsychotics, the embedded custom and practice of pro re nata (p.r.n. or 'as required') prescribing proved to be resistant to educational change interventions, ${ }^{1}$ whereas for the QIP targeting biochemical monitoring of lithium treatment, a major obstacle was the complexity of clinical care arrangements, including multiple interfaces between clinical and laboratory services, which were often not directly or wholly under the control of clinical teams. ${ }^{6}$

Thomas R. E. Barnes, MD, FRCPsych, DSC, Carol Paton, BSC, DipClinPharm,

FCMHP, Centre for Mental Health, Imperial College, Charing Cross Campus, London, and Prescribing Observatory for Mental Health, Centre for Quality Improvement, Royal College of Psychiatrists, London, UK

Correspondence: Thomas R. E. Barnes, Centre for Mental Health, Imperial College, 37 Claybrook Road, London W6 8RP, UK. Email: t.r.barnes@ imperial.ac.uk

First received 6 Jan 2012, final revision 23 July 2012, accepted 13 Aug 2012

\section{References}

1 Paton C, Barnes TRE, Cavanagh M-R, Taylor D, Lelliott P. High-dose and combination antipsychotic prescribing in acute adult wards in the UK: the challenges posed by p.r.n. prescribing. Br J Psychiatry 2008; 192: 435-9.

2 Barnes TRE, Paton C, Cavanagh M-R, Hancock E, Taylor DM. A UK audit of screening for the metabolic side effects of antipsychotics in community patients. Schizophr Bull 2007; 33: 1397-401.

3 Barnes TRE, Paton C, Hancock E, Cavanagh MR, Taylor D, Lelliott P, et al. Screening for the metabolic syndrome in community psychiatric patients prescribed antipsychotics: a quality improvement programme. Acta Psychiatr Scand 2008; 118: 26-33.

4 Paton C, Flynn A, Shingleton-Smith A, Macintyre S, Bhaumik S, Rasmussen J, et al. Nature and quality of antipsychotic prescribing practice in UK psychiatry of learning disability services: findings of a national audit. J Intellec Disabil Res 2011; 55: 665-74.

5 Perecherla S, Paton C, Shingleton-Smith A, Barnes TRE. Variations in antidementia drug prescribing. Prog Neurol Psychiatry 2009; 13: 30-3.

6 Collins N, Barnes TRE, Shingleton-Smith A, Gerrett D, Paton C. Standards of lithium monitoring in mental health trusts in the UK. BMC Psychiatry 2010; 10: 80

7 Paton C, Barnes TRE, Shingleton-Smith A, McAllister-Williams RH, Kirkbride J, Jones PB, et al. Lithium in bipolar and other affective disorders: prescribing practice in the UK. J Psychopharmacol 2010; 24: 1739-46.

8 Paton C, McIntyre S, Bhatti SF, Shingleton-Smith A, Gray R, Gerrett D, et al. Medicines reconciliation on admission to inpatient psychiatric care: findings from a UK quality improvement programme. Ther Adv Psychopharmacol 2011; 4: 101-10

9 Raleigh VS, Foot C. Getting the Measure of Quality: Opportunities and Challenges. The King's Fund, 2010.

10 Barnes TRE, Shingleton-Smith A, Paton C. Antipsychotic long-acting injections: prescribing practice in the UK. Br J Psychiatry 2009; 195: s37-42.

11 National Patient Safety Agency. Lithium Therapy: Important Information for Patients. NPSA, 2009. 